\title{
Proteinuria in rheumatoid arthritis—drug-induced or amyloid?
}

\author{
B. E. BOURKE, D. F. WOODROW, AND J. T. SCOTT \\ From Charing Cross Hospital, London W6
}

SUMMARY Over a 5-year period 325 patients with rheumatoid arthritis received treatment with gold (162) or D-penicillamine (163). Thirty-eight (11.7\%) developed proteinuria, which persisted in 10 patients; renal biopsies in these 10 patients revealed 6 cases of amyloid disease. These 6 cases illustrate the importance of investigation of rheumatoid patients developing proteinuria even when they are receiving potentially nephrotoxic drugs.

Secondary amyloidosis is a well known complication of rheumatoid arthritis, usually coming to attention when proteinuria or impaired renal function are noticed. Reports of its incidence vary with the selection of patients and the methods of detection employed. Arapakis and Tribe ${ }^{1}$ found a $5 \%$ incidence of amyloid on rectal biopsy in an unselected group of patients with rheumatoid disease of greater than 3 years' duration. Malstrom ${ }^{2}$ found amyloid in $19 \%$ of specimens of periapical dental tissue from another unselected group of rheumatoid patients. Missen and Taylor, $^{3}$ reviewing several collected series of necropsy studies on rheumatoid patients, found a $14 \%$ incidence of renal involvement. In all these studies the diagnosis was made by Congo red staining, with further confirmation from thioflavine $T$ or methyl violet. The usual sites for biopsy are kidney, liver, gingiva, or rectal mucosa, while Westermark and Stenkvist ${ }^{4}$ have successfully used subcutaneous fat aspirates to diagnose secondary amyloidosis.

Proteinuria in rheumatoid arthritis, however, also commonly occurs as a complication of gold or Dpenicillamine therapy. We report our experience over a 5-year period in patients with rheumatoid arthritis receiving antirheumatic therapy with gold or penicillamine under hospital supervision.

\section{Patients and methods}

A retrospective analysis was carried out from the records of all patients with rheumatoid arthritis

Accepted for publication 10 July 1980.

Correspondence to Dr J. T. Scott, Kennedy Institute of Rheumatology, Bute Gardens, London W6 7DW. undergoing supervision of therapy with gold or penicillamine at Charing Cross Hospital between 1974 and 1979. During this 5-year period 162 patients received gold and 163 patients received Dpenicillamine, the individual duration of therapy varying between 2 months and 5 years. Although the individual dosage schedule was controlled independently by the supervising physician, those patients receiving gold were usually treated with intramuscular injections of sodium aurothiomalate $50 \mathrm{mg}$ weekly up to a total dose of $1 \mathrm{~g}$ or less if clinical remission was achieved earlier, and thereafter reduced to a maintenance dosage of 20 to $50 \mathrm{mg}$ monthly. In the case of penicillamine therapy patients were usually started on $125 \mathrm{mg}$ of the drug daily, with $125 \mathrm{mg}$ increments at fortnightly intervals up to a maximum dose of $750 \mathrm{mg}$ per day, although the majority received a maximal dose of $500 \mathrm{mg}$ per day. All patients receiving gold or penicillamine had routine urine analysis performed at intervals varying from 1 week to 1 month. When proteinuria was detected by stick testing, quantitative estimations were performed by the standard reaction with biuret reagent (an alkaline $0.02 \%$ cupric sulphate solution). Drug therapy with gold or penicillamine was modified by the supervising clinician, but in most cases uncomplicated proteinuria of greater than $1.0 \mathrm{~g}$ per 24 hours was considered to be a sufficient reason in itself for withdrawal of the drug, at least temporarily.

Renal biopsies were performed under local anaesthetic with the patient in the prone position, under image intensifier control, specimens being obtained with a Tru-cut disposable biopsy needle. Biopsy specimens obtained from patients were 
processed and examined by both light and electron microscopy and by direct immunofluorescence as previously described..$^{5}$ In all cases the investigation for amyloid consisted of Congo red staining with apple green birefringence in polarised light, ultraviolet fluorescence with thioflavine $T$, and ultrastructural examination to demonstrate the presence of extracellular, randomly arranged, fine nonbranching filaments of 8-10 $\mathrm{nm}$ in diameter.

At the time of biopsy serum and urinary protein, blood urea, and plasma creatinine were estimated in the routine chemical pathology laboratories by AutoAnalyzer techniques.

\section{Results}

Thirty-eight patients $(11.7 \%)$ out of the total 325 patients included in the study developed proteinuria; 23 were receiving penicillamine and 15 gold. The dosage and duration of therapy were not significantly different in these patients from the group as a whole.

Initially the proteinuria was attributed to the therapy, but in the case of 10 patients it was persistent and in some associated with evidence of diminished glomerular filtration rate. Renal biopsies were therefore undertaken at periods ranging from two to 36 months after the development of proteinuria and the cessation of therapy. The clinical features of the 10 patients in whom biopsies were performed are summarised in Table 1. At the time of biopsy all but 1 had protinuria of greater than 1 $g$ per 24 hours, the exception being patient 4 , who had considerable elevation of plasma creatinine and blood urea, and in whom there was a long history of chronic urinary infection and impaired renal function. Two other patients at the time of biopsy had raised levels of blood urea and plasma creatinine (9 and 10).

\section{RENAL BIOPSY FINDINGS}

Six of the 10 patients were found to have amyloidosis, confirmed in all by Congo red and thioflavin $T$ staining. In 5 of these 6 patients electron microscopy was also confirmatory (Fig. 1), while in the sixth case (patient 6), although there was increased mesangial matrix, no filamentous amyloid could be demonstrated. By light microscopy amyloid deposits were found in the glomeruli of all 6 patients and in the interstitium and arteriolar walls of 3 patients.

Of the 4 remaining patients 3 had evidence of an immune complex glomerulonephritis $(1,2$, and 3$)$ with granular deposits of IgG, Clq, and C3 along the glomerular capillary loops. In 2 of these cases (1 and 3 ) the electron microscopic changes of small subepithelial electron dense deposits characteristic of an early membranous glomerulonephritis (Fig. 2), while in the third (patient 2), the lesion was difficult to classify, as the glomeruli were normal by light microscopy and no glomeruli were present in samples prepared for electron microscopy. The biopsy findings in the remaining patient (4) revealed evidence of a chronic interstitial nephritis; this patient had a known history of chronic urinary infections. In one patient (1) a urate tophus was found in the renal medulla, a surprising and presumably incidental finding.

The subsequent progress of the patients with amyloid is summarised in Table 2 , the maximum duration of follow-up being 4 years.

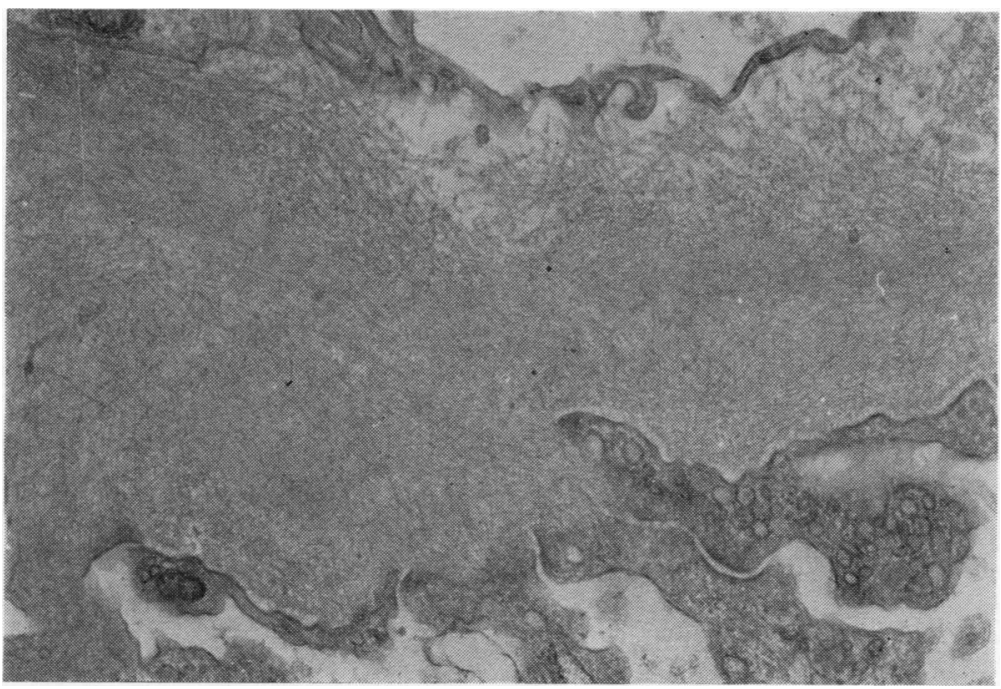

Fig. 1 Electron micrograph of thickened glomerular basement membrane showing the randomly arranged fine filaments of amyloid throughout its thickness. (Uranyl acetate, Reynolds lead citrate, $\times 16700)$ (patient 8). 

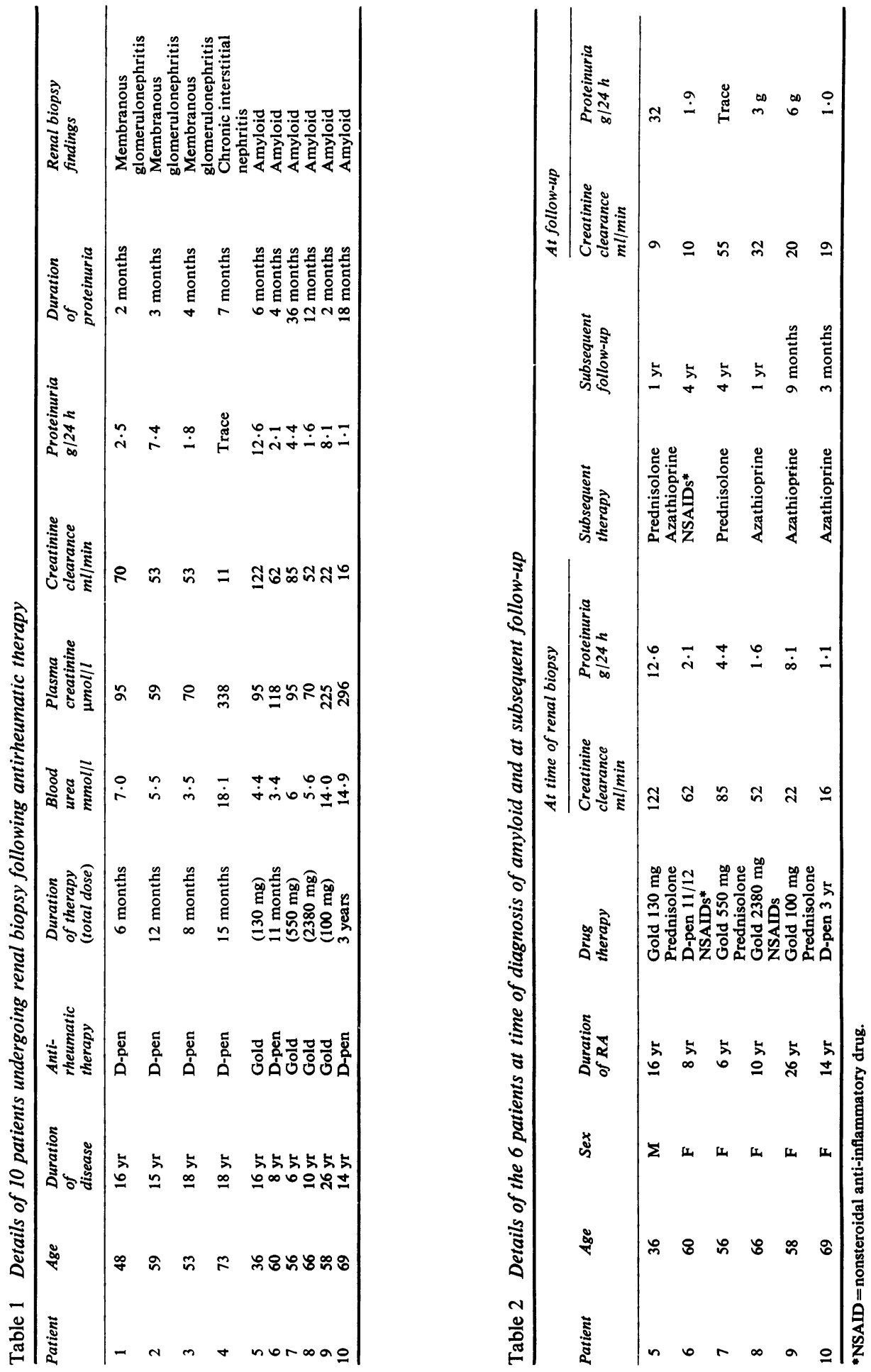


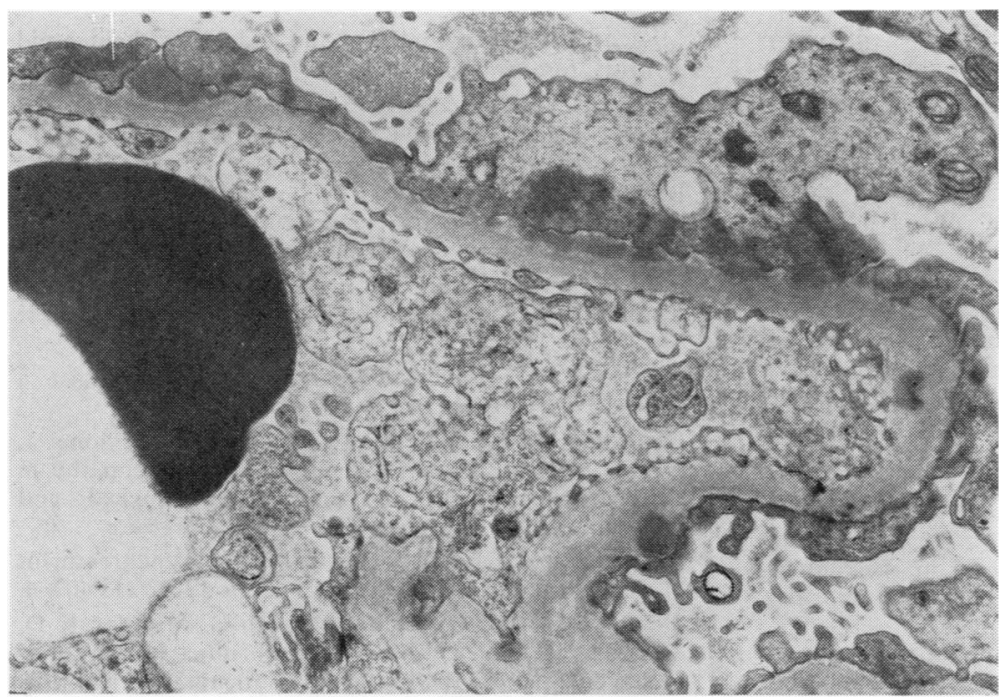

Fig. 2 Electron micrograph of a glomerular capillary loop showing small discrete subepithelial cell electron dense deposits within the basement membrane. (Uranyl acetate, Reynolds lead citrate. $\times 10400)$ (patient 1).

\section{Discussion}

The 6 cases of secondary amyloidosis came to light initially because frequent regular tests for proteinuria were being performed. The proteinuria was initially attributed to drug therapy, but its persistence after drug withdrawal led to subsequent investigation and ultimately to the diagnosis of amyloid. These 6 patients all had classical or definite rheumatoid arthritis without any history of tuberculous or other chronic infection apart from patient 8 , who had a history of an infected hip prosthesis lasting 6 months some 5 years prior to diagnosis of amyloid.

Secondary amyloidosis is a potentially fatal complication of rheumatoid arthritis, and is more likely to occur in severe or chronic forms of the disease. The course of amyloid is variable, but may be influenced by treatment of the primary disease. ${ }^{6}$ Patients 6 and 7, followed up over 4 years and treated only with prednisolone and nonsteroidal anti-inflammatory drugs, have developed mild deterioration of glomerular filtration rate, while proteinuria has diminished. The other 4 patients have been treated with azathioprine, but follow-up has been short; 1 of them rapidly developed severe renal failure (patient 5), another has shown a significant fall in glomerular filtration rate (8), and the remaining 2 patients $(9$ and 10 ) have maintained their already compromised renal function.

The relationship between the development of amyloid to the drugs is uncertain and probably coincidental. There is no evidence that gold or penicillamine lead to the production or deposition of amyloid; in fact if treatment of the underlying disease inhibits amyloid formation, it would be expected that these drugs would prevent rather than encourage secondary amyloid deposition.

In this series the incidence of proteinuria with penicillamine was $14.1 \%$, which is similar to that in other recent series. ${ }^{78}$ The incidence with gold was $9.2 \%$, again in accordance with other series. Three of the 10 patients with persistent proteinuria had an immune complex glomerulonephritis consistent with a penicillamine induced nephropathy. Drug induced proteinuria may persist for a variable time after cessation of gold or penicillamine, and durations of 1 year or more are reported with the latter drug. ${ }^{79}$ However, in view of the findings reported here, it is clear that the possibility of amyloid should be considered if proteinuria in these patients does not disappear within, say, 3 months. Also of importance was evidence of considerably impaired glomerular filtration rate in some of the amyloid patients when proteinuria was first noted. This feature is not normally associated with gold of D-penicillamine induced nephropathy, and therefore appears to favour the diagnosis of amyloid.

In the series of Bacon et al. ${ }^{8}$ no cases of amyloid were found on renal biopsy in 14 patients who developed proteinuria on penicillamine, but in a recent series Stockman et al. ${ }^{10}$ found 1 case of renal amyloid from 6 patients on penicillamine undergoing renal biopsy for proteinuria.

Over the 5-year period of this study only 1 other case of amyloidosis complicating rheumatoid arthritis came to light. It seems therefore that regular urine testing performed because of the known nephrotoxic effects of these drugs was the 
most important factor leading to the ultimate diagnosis of amyloid and stresses the importance of urine testing in all severe or chronic rheumatoid patients. This study also underlies the importance of early investigation of rheumatoid patients developing proteinuria even while on treatment with nephrotoxic drugs.

We are grateful to members of the Renal Unit at Charing Cross Hospital for their collaboration in the assessment of these patients and for performing the renal biopsies, to Mrs Jill Moss for the electron microscope studies, and to $\mathrm{Dr}$ R N Maini for permission to report some of these patients who were under his care.

\section{References}

1 Arapakis G, Tribe C R. Amyloidosis in rheumatoid arthritis investigation by means of rectal biopsy. Ann Rheum Dis 1963; 22 : 256-62.

2 Malstrom M. The nature of the inflammatory process in the teeth supporting tissues of patients with rheumatoid disease. Scand J Rheumatol 1975; Suppl 6: 1-28.
3 Missen G A K, Taylor J D. Amyloidosis in rheumatoid arthritis. J Pathol Bacteriol 1956; 71 : 179-92.

4 Westermark P, Stenkvist B. A new method for the diagnosis of systemic amyloidosis. Arch Intern Med 1973; 132: 522-3.

5 Rainford D J, Woodrow D F, Sloper J C, de Wardener H E, Griffiths I. Post meningococcal acute glomerular nephritis. Clin Nephrol 1978; 9: 249-53.

6 Parkins R A, Bywaters E G L. Regression of amyloidosis

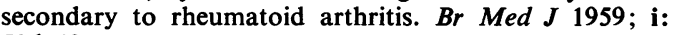
536-40.

7 Huskisson E C. Penicillamine and the rheumatologist: a review. Pharmatherapeutica 1976; 1 : 24-39.

8 Bacon P A, Tribe C R, Mackenzie J C, Verrier Jones J, Cumming R H, Amer B. Penicillamine nephropathy in rheumatoid arthritis. A clinical, pathological and immunological study. $Q J$ Med 1976; 45: 661-84.

9 Huskisson E C, Hart F D. Penicillamine in the treatment of rheumatoid arthritis. Ann Rheum Dis 1972; 21 : 402-4.

10 Stockman A, Richter D, Barraclough D, Muirden K D, Macdonald I, Kincaid-Smith P. Difficulties in the use of D-penicillamine in the treatment of rheumatoid arthritis. Aust NZ J Med 1979; 9: 495-503. 